\title{
openemotionmap.org - Emotional response to space as an additional concept in cartography
}

\author{
Georg Gartner \\ Institute of Geoinformation and Cartography, Vienna University of Technology, \\ Erzherzog-Johannplatz 1, A-1040 Vienna, Austria \\ georg.gartner@tuwien.ac.at
}

KEY WORDS: Cartography, Location-based Services, sensing emotions

\begin{abstract}
:
In this submission the concept is analysed, that the emotional relation and the degree of emotional response is structuring space and can therefore be relevant for human wayfinding. Possible measures for linking explicit links between person's memory/ experience/ emotional response with the physical environment are investigated and possible applications for navigation systems are discussed.
\end{abstract}

\section{INTRODUCTION}

The rapid development of the mobile internet enforces the emergence of location based services. In addition to the more familiar car navigation systems, guiding systems for pedestrians are gaining in importance. In this context, the integration of attributes of spatial objects, such as landmarks, are widely accepted as being a necessary component of guiding systems; however, it is not entirely clear how the main aspects of space should be modeled. This paper provides a new approach based on psychological and geographic literature to further our understanding of navigation and way finding in ubiquitous environments, and introduces the concept of the subjective or emotional element of space.

Imparting spatial information usually involves cartographic presentation forms. Ubiquitous cartography methods including Location Based Services (LBS) can be seen as enabling new forms of cartographic communication processes. Navigation of our macro environment is a human ability that we tend to take for granted unless it fails. For example, we may get lost when visiting a new city. To find our way, we utilize various strategies such as relying on maps/technology created by others or by asking directions (other referent), or by searching using a specific landmark as a referent point (self-referent). As we become more familiar with the landscape, we build our own cognitive map of the area and subsequently rely on and use it to find our way. Once mentally constructed, we may add to or update our cognitive map, but generally the initial map appears to be relatively permanent and can serve as an important way-finding reference.

Lack of conscious awareness of the processes involved when performing tasks that activate and/or execute automated navigation and way finding processes, raises questions about the role that subjective evaluations and emotions play in the development, retention, and use of navigation/way-finding maps. That is, immediate subjective feelings about--and therefore emotional associations with - space and especially landmarks, hence emotional landmarks, may be tied automatically to the cognitive processes associated with navigation and way finding. Conse- quently, an emotional representation may enhance or facilitate cognitive mapping.

In order to explore this concept, in this paper a first review on existing approaches on conceptualizing emotional geographies and methods of sensing emotions is reviewed. This provides the basis for the argument that emotional responses to space are highly relevant as an aid in navigating our environment. The article concludes with an evaluation of the relevance for investigating the role of emotional response to space in navigation and way finding.

\section{SENSING EMOTIONS}

Various methodologies have been tested and developed for sensing emotions, which is understood in this context as one of the psychological processes (perception, cognition, memory, emotion, behaviour, physiology) that devices and sensors can 'experience' from (Westerink 2008). Different emotional expressions (or states of emotions) have been identified in the context of sensoring, among them are anger, sadness, happiness, cheerfulness (Tosa et al 1994); neutral, joy, boredom, sadness, anger, fear, indignation (Mozziconacci 1995); anger, fear, sadness, joy, disgust (Scherer 1995); neutral, happiness, sadness, anger, fear, boredom, disgust (Scherer 1995) and fear, anger, sadness, happiness (McGilloway et al 1995).

Several standard procedures exist for measuring the experience-related processes (Westerink et al 2008):

a. judgements \& questionnaires (often elicited from the user, e.g., self-reports in the form of interviews or questionnaires)

b. behavioural observations

c. physiology recordings: e.g., ECG (electrocardiogram), EMG (electromyogram), GSR (galvanic skin response), BVP (photoplethysmograph)

The above measurements can also be automated to a certain extent, each type requiring a certain time period to come to relevant interpretation: For judgements \& questionnaires this period is estimated to be several minutes, while for behaviour observations, it is more likely to be in the order of seconds and 
for physiology recordings events might already get noticed over the time course of several milliseconds. Jennifer A. Healey made a comparison on different physiology recordings (ECG, EMG, GSR, BVR) in (Healey 2008). Martin Ouwerkerk suggested using unobtrusive/unnoticeable sensing for physiology recordings. The key technology of this is "miniature wireless sensors" (Ouwekerk et al 2008).

For products and services, the emotional qualities of experiences are very important. For services as tools (which are meant to save time) positive experiences are seen as an asset, whereas negative experience should be avoided. Typical leisure time services (which are meant to spend time on), on the other hand, are often intended to deliver emotions, positive and negative alike. A wayfinding service can be seen as somehow inbetween the function of leisure time services and tool services.

Three types of measurement environments have been used and tested (Healey 2008) so far for sensing emotions, including a laboratory setting, an ambulatory setting and an automobile szenario. The challenges for eliciting affective responses include:

a. The main challenge with measuring affective response in the laboratory is generating authentic affective responses within the short time the subjects participate and within the ethical guidelines of most oversight committees on the use of human subjects.

b. The two greatest challenges in the ambulatory environment are differentiating affective physiological responses from other physiological responses and accurately capturing affective ground truth.

c. The automobile provides a compromise situation where the subject has restrained movement and where the situational ground truth can be recorded but where the subject can also face genuinely dangerous and unexpected situations. Although the range of emotions experienced while driving can be limited, the strength of feeling is often genuine and strong.

There are not many reported applications about making use of sensored emotions available. Mincheol Whang gave an example of "emotional computer" (2008), and Ben Mulder et al. used "Physiological Measures" for task adaptation (2008).

\section{EMOTIONAL WAYFINDING}

The emotional significance of a particular space may enhance its remembrance and increase the accuracy of direction decisions. Despite the strong component of internal decision making and memory in wayfinding, however, no psychological literature was found that specifically addressed the facilitative significance of emotional landmarks in the context of every day wayfinding and navigation. Instead, references in the psychological literature pertaining to emotional landmarks are often vague, ill defined, or applied in a different context. Articles reviewed were generally centered on early childhood emotional development/ developmental delay, emotional trauma, end-oflife developmental landmarks. Thus, any reference to emotional geographies is usually discussed within the context of inner personal growth arising from a traumatic event. Other researchers have focused on understanding the cognitive representations of a person's perception of a spatial environment through cognitive maps. Cognitive mapping deals with the underlying psychological structure of the environment as well as the distance between places. Thus, the psychological literature has drawn freely from geographic terminology as a way of articulating the internal navigational process(es) associated with emotional growth or cognitive representations, whereas the geographical literature has focused predominantly on navigating and wayfinding in the external, physical environment. Although neither of these literature areas investigated the role of emotional landmarks as an aid to navigating the external physical (or virtual) environment directly, much can be learned from a brief review as research in both areas attempt to understand the inner navigation process and/or inner spatial representation of the individual. Currently, the role that emotion plays in facilitating way finding and navigation has been difficult to examine. Lack of research geared toward the development of appropriate methodologies may stem partially from cultural biases toward favouring logic or cognitive strategies and denying or devaluing the role(s) of emotion.

The measurement of unconscious awareness has had limited success to date, perhaps because the study of consciousness was until recently viewed as "unsuitable for scientific research" (Zahavi, 2004) and regarded with suspicion (Damasio, 1999). A recent increase in the literature suggests there is a resurgence of interest in this topic likely due to changes in technological development and conceptual changes (Zahavi, 2004). Instead, an interesting paper by Merskey (1997)--in which he struggled to define consciousness in the context of pain and behaviour-appears to be quite relevant to the current topic. He identified three elements of consciousness: (1) the occurrence of an observable external event accompanied by an experience (i.e., sensation or emotion--with or without an external reference point); (2) an internal emotional state, and (3) an awareness of knowing something. Merskey further stated that, in the first element, there may or may not be some sort of external reference point. This particular definition of consciousness may provide an important starting point for research investigating emotional landmarks in the real or virtual world. For example, it is proposed here, that a landmark may include both an internal and an external reference point--connected via both an individual's emotional state as well as his or her cognitions. This idea is consistent with Goldie's (2000) classification of feelings as internally focused (i.e., feelings related to one's own bodily sensations) versus externally focused (i.e., feelings related to an object). It is also proposed here that the third element identified by Merskey - awareness of knowing something - may serve as the mechanism for obtaining information about emotional landmarks.

The utility of an emotional response to space does not minimize or eliminate the relevance of building a cognitive map of an environment. Rather, an emotional landmark is thought to enhance (positively or negatively) the ability to develop a cognitive map; emotional processing is presumed to be automatic and without conscious choice. The ability to link the emotion to a specific event at a specific landmark may facilitate not only way finding and navigation during times when one's cognitive state is overloaded, but it may actually speed up the every day process of cognitive mapping. Such an exploration may also help elucidate individual differences in the self-estimation of spatial anxiety and environmental competencies that were discussed by Schmitz (1999), as well as reveal how emotional landmarks relate to landmark and/or route finding preferences. Although not stated, the primary and secondary types of survey knowledge reported by Thorndyke and Hayes-Roth (1982) which are acquired through immediate interaction with the environment would, presumably, be linked with the emotional and sensory state of the explorer. An examination of the role of emotional landmarks, therefore, may contribute to our knowledge of navigation and way finding in the physical world, in cyberspace in general, and cybercartography in particular. Although not specifically referred to as emotional landmarks, the ideas discussed above are consistent with the conceptual 
model of the environment-behaviour interaction developed by Kitchin (1996) which suggests that knowledge acquisition of the environment is derived through emotionally biased memoryprocessing systems.

\section{CONCEPTUAL STRUCTURE}

Emotional response to space can be characterized by its emotional significance for an individual, wheras a physical connection to the environment does not exist. The individual moulding of an objects' emotional significance can be further classified to direct-, indirect- and collaborative response according to the form and status of physiological development (Davidson et al 2006).

Direct responses to space or spatial objects are created by direct episodic experiences, thus form direct episodic memories. The dependency on time is source for further determination to "history based episodes", which bases on individual experiences in the past, and "current episodes", where current emotions and experiences build up new landmarks within the personal knowledge base.

Indirect landmarks are built up by "third party events", which form some kind of semantic memory based on third party narrations. The creation of indirect semantic landmarks may be achieved by personal narrations of others, where variations of emotional response depending on the basis of relationconfidence are expectable, or factual knowledge extracted from different kind of media, like books, data, news, etc..

Collaborative responses to space are the result of demographic reasoning. Although this class seems to consist of an external, thus non individual, component, this is the result of stochastic evaluation. Actually no physical obvious relations, but physiological accordances among the user-group to specific objects or behaviors may be observable. The result of these observations describes social landmarks, which should have strong relations to individual episodes and the internal knowledge of the individual.

In order to serve as additional "layer" or landmark for navigation tasks each of these classes need to have a link to space. This reference to the external, real environment may be in form of a direct or indirect connection to or description of space with various granularities and degree of emotional response. As next step an empirical verification of the concept with various user groups and different scenarios is aimed at.

\section{5. openemotionalmap.org}

Environmental factors, such as safety, convenience, and attractiveness, are relevant for the perceived route quality in pedestrian navigation (Schmidt et al., 2011).

Although these factors are not emotions itself, they are affective evaluations caused by the environment. In a current project at Vienna University of Technology ("EmoMap-Project"), the aim is to gather emotional responses evoked by the environment. These emotional responses to space will be therefore modelled as a person's evaluation of affective qualities. The methodolody consists of user-centered approaches including user evaluations of the environment by ratings. We expect the collected data to be - at least to some extent - "cleaned" from non-spatial feelings of the person. According to Russell (2003) affective qualities are routinely evaluated anytime. We therefore expect to collect data, which is easily reportable by anyone, and present at any time. For gathering this affective information, we will use a crowd sourcing approach to get a high volume of data.

Self reports are the most promising method, since people can simply and freely contribute via their own mobile application any time and anywhere. People's perception of affective quality will be measured by implementing a Semantic Differential (SD), invented by Osgood et al. (1957), into a mobile application.

After implementing the preliminary emotion model into a prototypical mobile application, the usability, as well as the emotion and location model is currently examined by using the application in the study area of Vienna. First results from people's affective responses to space are analysed currently. Preliminary results from user's responses and correlations between the parameters suggest to aggregate, and further reduce the number of parameters.

\section{CONCLUSIONS}

The inclusion of emotional layers and landmarks in multimedia, multi-sensory ubiquitous cartographic technologies, such as LBS, is expected to provide a deeper understanding of navigation, way finding and conceptualizing of space. This knowledge will provide direction for the selection of content use in development of ubiquitous cartographic supports for both the real and virtual worlds. That is, this knowledge may lead to both the development of augmentation devices for those who have difficulty in way finding, as well as a means of enhancing the experience for the user.

\section{References}

Damasio, A., 1999. The Feeling of What Happens. San Diego, CA: Harcourt.

Davidson J., Smith M., Bondi L., 2006. Emotional Geographies. Hampshire.

Goldie, P., 2000. The Emotions: A Philosophical Exploration. Oxford: Clarendon.

Healey, Jennifer A., 2008. Sensing Affective Experience. In: Westerink et al (eds) Probing Experience From Assessment of User Emotions and Behaviour to Development of Products, Springer.

Kitchin, R. M., 1996. Increasing the Integrity of Cognitive Mapping Research: Appraising Conceptual Schemata of Environment-Behaviour Interaction. Progress in Human Geography 20, pp. 56-84.

McGilloway, S., Cowie R., Cowie E.D., 1995. Prosodic signs of emotion in speech: preliminary results from a new technique for automatic statistical analysis. ICPhS, Vol. 1, pp. 250.

Merskey, H., 1997. Pain, Consciousness and Behaviour. Pain Research and Management 2, pp. 118-122.

Mozziconacci, S., 1995. Pitch variations and emotions in speech. ICPhS Vol. 1, pp. 178.

Mulder Ben et al,. 2008. Using Physiological Measures for Task Adaptation. In: Westerink J. et al (eds) Probing Experience From Assessment of User Emotions and Behaviour to Development of Products, Springer.

Osgood, C.E., et al., 1957. The Measurement of Meaning. Urbana: University of Illinois Press.

Ouwerkerk, M., Pasveer F., Langereis G., 2008. Unobtrusive Sensing of Psychophysiological Parameters. In: Westerink J. et al (eds) Probing Experience From Assessment of User 
Emotions and Behaviour to Development of Products, Springer.

Russell, J.A., 2003. Core affect and the psychological construction of emotion. Psychological Review, Vol. 110 Issue 1, pp. 145-172.

Scherer, K.R., 1995. How emotion is expressed in speech and singing. ICPhS Vol. 3, pp. 90.

Schmidt, M., Gartner, G., Steinmann, R., Häusler, E., 2011. Using Subjective Route Qualities to Enhance Pedestrian Route Planning, Proceedings of the 25th International Cartographic Conference, Paris. 2011.

Schmitz, S., 1999. Gender Differences in Acquisition of Environmental Knowledge Related to Wayfinding Behavior, Spatial Anxiety and Self-Estimated Environmental Competencies. Sex Roles 41, no. 112, pp. 71-93.

Thorndyke, P. W., Hayes-Roth B., 1982. Differences in Spatial Knowledge Acquired from Maps and Navigation. Cognitive Psychology 280, pp. 560-589.

Tosa, N. et al., 1994. Neuro-Character, A AAl'94 Workshop, AI and A-Life and Entertainment.

Westerink J., 2008. Experience in products. In: Westerink et al (eds) Probing Experience From Assessment of User Emotions and Behaviour to Development of Products, Springer.

Westerink J. et al., 2008. Introduction: probing experience. In: Westerink J. et al (eds) Probing Experience From Assessment of User Emotions and Behaviour to Development of Products, Springer.

Whang, M., 2008. The Emotional Computer Adaptive to Human Emotion. In: Westerink J. et al (eds) Probing Experience From Assessment of User Emotions and Behaviour to Development of Products, Springer.

Zahavi, D. 2004. Editorial Introduction: The Study of Consciousness and the Reinvention of the Wheel. Journal of Consciousness Studies 11, no. 10-11, pp. iv-viii. 